\title{
Path to Global Accounting Convergence
}

\author{
Ariet Malaj \\ Professional Academy of Business, Tirana, Albania
}

\section{Doi:10.5901/ajis.2015.v4n3p53}

\begin{abstract}
Increasing globalization of economical enterprises activities, as well as internationalization of financial markets has increased the need to harmonize accounting standards. It is not appropriate for these markets to have diversity of standards, according to which financial statements on accounting basis are prepared and introduced, for the diversity makes it difficult for financial analysis and as a consequence, it causes difficulty in assessing performances and financial positions of different enterprises, whom titles are shown in a given market. For investors, capital shareholders, lenders, suppliers and also employees and the general public, this diversity is considered as an obstacle for decision-making, especially for those related to the delivery of economical sources in different enterprises. Concerning the above, EU issued Directives IV, related to annual accounts, where there are included accounting balance, statement and profit, as well as notes on charge accounts. These documents are part of the whole and they must be designed clearly and in accordance with the recommendations of Directives IV, in order to provide a full and accurate view of activities, obligations, capitals and profiting or losses of companies in the period they part of. While, under the perspective of Directives VII, thememeber states of EU make any enterprise, under their jurisdiction, stand before obligations, in order to prepare consolidated accounts and a solid report of direction, where each case the accounts of mother or daughter companies shall be consolidated near mother companies, besides the location of daughter companies. On the other hand, lacking planned information according to same standards, the market cannot function effectively and reliably. Thus, this is a basic argument for the need and benefits of different enterprises, whatever their place of origin is, they shall supply intruders in the capital markets with reports or financial statements prepared and introduced according to the same accounting standards. If there is more pressure on investors and lenders have a better protection when they are given financial information prepared according to high quality standards and which normally ensure transparence and reliability, then it is known why main international bodies, since a long time, they have urged all countries to implement the new standards of accounting, on basis of compliance with international ones.
\end{abstract}

Keywords: Globalization, Directives, Convergence, financial statement, consolidated accounts, accounting standards

\section{Introduzione}

Il progresso professionale e tecnologico e il trasferimento piu' veloce dell'informazione, delle persone, dei beni e servizi hanno portato il mondo piu' vicino. La crescita del commercio internazionale e i flussi del capitale hanno stimolato una sempre piu' crescente integrazione economica. Come conseguenza di questi sviluppi c'e' stato un effetto internazionale su piu' usi, pratiche e istituzioni. Nella vita dell'imprenditoria questo ha portato, soprattutto, al desiderio di regolare ed armonizzare gli Standards della Contabilita' tra gli Stati, come pure la necessita' della loro internazionalizzazione per le piccole e medie imprese.

L'Albania si trova sotto l'effetto di una serie di riforme e cambiamenti economici che la preparano per il suo ingresso nella U.E.. Con la speranza, che noi in un futuro, non lontano, saremmo parte dell'economia comune europea, allora lo scopo di questo articolo e' di evidenziare il piu' possibile in maniera chiara, il livello dell'applicazione degli Standards Nazionali della Contabilita', come pure il rapporto importante esistente tra la contabilita' con gli standards e la professione contabile in Albania. Valutare la compatibilita' e il livello necessario delle cognizioni per la professione contabile. Valutare il processo e l'applicabilita' della Contabilita' e dei suoi Standards, in concomitanza con la base legale locale, le richieste dell'integrazione e della globalizzazione, come un bisogno del tempo per assicurarsi l'integrazione nella U.E..

\section{Metodologia}

Secondo (Welman e Kruger 1999), la raccolta dei dati qualitativi e quantitativi vanno fatti in funzione alla realizzazione e alla proiezione verso un approccio di studio. I dati utilizzati in funzione dell'analisi dei problemi dell'applicazione degli 
standards nel nostro paese, in particolar modo sono qualitativi (connessi alle caratteristiche dei gruppi similari) e quantitativi (per rendere possibile l'analisi empirica). Questi dati vengono raggruppati ulteriormente in dati di prima rilevanza e di seconda rilevanza. L'assicurazione dei dati primari, e' stato reso possibile grazie ai questionari semplici. Mentre i dati secondari, sono stati assicurati all'Istituto degli Esperti Contabili Autorizzati (IEKA) (un importante istituto nel campo della contabilita'). Attraverso le analisi statistiche descrittive, conoscitive e comparative, come pure tramite il test dei dati, si rende possibile capire la tendenza dello sviluppo della pratica per le professioni contabili e la via del processo dell'armonizzazione, ed anche seguire le raccomandazioni necessarie allo scopo dell'applicazione il piu' efficacemente possibile degli standards della contabilita' nel nostro paese.

\section{La Regolazione Contabile a Livello Europeo, Bisogno e Meta del Futuro}

L'armonizzazione contabile a livello delle Comunità Europea ha alla sua base il trattato di Roma del Marzo 1957, che si sarebbe realizzata attraverso il coordinamento della legislazione contabile nazionale degli Stati membri. Per la prima volta la necessità di questo coordinamento è stato pubblicato nella gazzetta ufficiale della Comunità europea del marzo 1978, il Consiglio della U.E. ha approvato la quarta direttiva.

Nel campo dell'applicazione della quarta direttiva entrano i prospetti finanziari (i conti annuali) individuali della società, qualificate società del capitale (societa azionarie, s.p.a.) come alcuni tipi delle altre società collettive, semplici società ecc.

La quarta direttiva accentuava specialmente:

- La necessità e l'urgenza di un coordinamento contemporaneo nel campo della contabilità per le società succitate, a causa che, da un lato, l'attività economica di queste società si estende oltre i confini del territorio nazionale e che, dall'altro lato queste società offrono come garanzia per i terzi solo il patrimonio della stessa società.

- La necessità per decidere in comunità le condizioni giuridiche minime quanto alla diffusione dell'informazione finanziaria, che va reso noto al pubblico da parte delle società concorrenti.

- L'importanza del coordinamento dei vari metodi della valutazione per assicurare, che l'informazione finanziaria sia comparabile ed equivalente, che viene rispecchiata nei prospetti finanziari.

Nella presentazione dei motivi del coordinamento delle disposizioni nazionali, quanto alla preparazione e alla presentazione dei prospetti finanziari, questa direttiva insiste specialmente nell'importanza del suo coordinamento quanto alla tutela degli azionari (soci) e di terzi.

Come è stato precedentemente detto, la quarta Direttiva aveva a che fare con i prospetti finanziari individuali. Ma un gran numero di società facevano parte del gruppo delle società,

dalle quali era necessario, che preparassero prospetti finanziari consolidati, allo scopo che ai soci e ai terzi fosse resa nota l'informazione finanziaria per l'insieme delle società del gruppo, come se si trattasse di una sola società.

Le direttive sono previste, che in un prossimo coordinamento gli Stati membri possono non applicarla per le banche e le altre isitituzioni finanziarie e per le società assicurative. Per le società assicurative è stato pubblicato nel 1989 una speciale direttiva (la Direttiva nr. 89|177|CFF)

Per fare sì, che l'informazione finanziaria sia comparabile ed equivalente è importante un secondo atto di coordinamento, che è stato concretizzato con l'approvazione il 13 giugno 1983 della settima Direttiva del Consiglio della Comunità Europea. Tutte e due queste direttive sono state adottate nella legislazione degli Stati membri, chi più velocemente, chi meno. La seguente tabella illustra l'anno dell'adozione e l'anno dell'inizio dell'applicazione delle direttive secondo alcuni Stati membri.

\begin{tabular}{lcccc} 
& \multicolumn{2}{c}{ La quarta Direttiva } & \multicolumn{2}{c}{ La settima Direttiva } \\
Gli Stati membri & L'adozione & Applicazione & L'adozione & Applicazione \\
Belgio & 1983 & 1984 & 1986 & 1990 \\
Francia & 1983 & 1984 & 1985 & 1990 \\
Italia & 1990 & 1993 & 1990 & 1994 \\
Germania & 1985 & 1986 & 1985 & 1990 \\
Olanda & 1983 & 1985 & 1988 & 1990 \\
Inghilterra & 1983 & 1985 & 1987 & 1989
\end{tabular}

Alcune caratteristiche principali dell'armonizzazione delle regole contabili in seno alla Comunità Europea sarebberero;

- L'armonizzazione europea ha seguito il modellamento delle società europee. 
- L'armonizzazione europea è stata ottenuta grazie agli strumenti giuridici, le direttive proposte dalla Commissione Europea approvate dal Consiglio e dal Parlamento Europeo. A questo punto gli Stati europei sono obbligati a incorporare nel diritto nazionale le regole basilari per l'informazione finanziaria.

- Le regole contenenti le direttive contabili sono regole indispensabili e gli Stati membri possono stabilire regole più complete e severe specialmente per le società quotate.

- Le direttive contabili europee non mirano alla standardizzazione dell'informazione finanziaria. La finalità dell'armonizzazione consiste nella preparazione dell'informazione pubblicata dalle società del capitale considerato equivalente e comparabile.

- Le direttive ammettono modifiche contabili variabili ( non le stesse ).

- Le direttive europee prevedono schemi a carattere obbligatorio in merito alla compilazione del bilancio, il rispecchiarsi del risultato e il contenuto minimo per i prospetti esplicativi. Per le società di poca rilevanza economica, vengono autorizzati schemi semplificati.

Le direttive contabili europee hanno aiutato visibilmente nel miglioramento della qualità dell'informazione finanziaria in seno ai paesi membri della comunità, pur non essendo stato completamente la finalità dell'essere comparabile. Questo a causa delle molteplici opzioni contenenti le direttive e delle interpretazioni divergenti fatte ai principi basilari, in essi raffigurate. L'esistenza delle opzioni nelle direttive contabili, come pure nel caso di IAS (International Accounting Standards), si originava per via dell'atteggiamento consensuale per la loro compilazione. Gli Stati membri avevano creato da tempo i loro sistemi contabili, prodotti, delle condizioni economiche, giuridiche e sociali di ciascun paese e frutto della convergenza dei punti di vista dei professionisti della contabilità e delle angolazioni dei governi legate agli obiettivi della contabilità.

\section{Le Direttive Contabili Europee Sotto la Pressione del Continuo Cambiamento}

Le direttive di U.E., per i prospetti finanziari, sono nati dalla necessità dell'armonizzazione e delle regole contabili a livello europeo, come risultato della globalizzazione dei rapporti economici del continente e più in là. Con l'aumento di questi rapporti e i processi integrativi del vecchio continente, anche le regole giuridiche dell'elaborazione della presentazione quanto all'informazione economica e finanziaria hanno subito modifiche e adattamenti continui mirando allo stesso tempo a una tutela migliore degli azionisti ed una espressione più unica e comparabile dell'informazione avuta dai sistemi contabili.

Così la quarta direttiva, approvata nel luglio 1978, seguendo la perfezione della legilazione dei paesi membri, ha subito più volte modifiche, rielaborazioni e aggiornamenti. La sua prima versione, edita nella gazzetta ufficiale della Comunità Europea il 14.8.1978 è stata ammendata, rielaborata ed edita nel 1979, 1980, 1982, 1983, 1985, 1989, $1990,1992,1994$ ecc.

La stessa sua fortuna ha avuto anche sua sorella, la settima Direttiva per i conti consolidati, che inizialmente è stata edita nella gazzetta ufficiale il 18.07.1983 ed è stata ammendata, rielaborata ed edita nel 1985,1988,1990,1993, ecc.

Nel momento attuale, quando l'Europa unita non è solo con un unico slogan di azione, ma un processo storico in via di consolidamento, dove la politica e l'economia stanno andando verso prognosi uniche ed interconnesse dello sviluppo, la necessità della perfezione e dell'adattamento dell'informazione contabile è diventata ancora più impellente. In questo ambito oggi in Europa c'è un ampio dibattito per modificare le direttive 4 e 7.

Le varianti di queste modifiche sono state messe in circolazioone per essere discusse, chi di più chi meno, mirano a un adattamento più fedele agli standars internazionali della contabilità. Tra le varianti in discussione, la più spiccata è la "versione dell'alto livello", presentata dalla federazione europea degli esperti contabili nell'aprile del 2001. Secondo questa variante le modifiche mirano a essere fatte in quegli articoli trattanti questioni, non toccate negli standards internazionali, negli standards della contabilità. Questa variante modificatrice delle direttive della contabilità intende le società, che utilizzano IAS, come conto dell'individuo e consolidati, in concomitanza con le regole approvate, oppure con la legge contabile di ogni paese, membro della U.E.

Una variante dell'alto livello sarebbe, che si includessero altre voci contenenti una base minima per il rapporto finanziario ( stabilire la sfera della direttiva, definire i conti annuali, la concezione del punto di vista onesto e vero), oltre le voci citate nella "versione dell'alto livello".

Nella versione dell'alto livello va trovata una soluzione per le altre società, che non utilizzano IAS. E' dubbio se si potesse arrivare a che fossero comparabili, se gli standars nazionali della contabilità si utilizzassero al posto degli standars internazionali della contabilità. Vari autori argomentano, che va fatta una intera modernizzazione delle direttive 
nel nostro contesto, le società le più probabili a essere toccate, sarebbero le medie e le piccole imprese. Qualche opinione ha suggerito, che si fosse inserita anche un'alternativa per le medie e le piccole imprese utilizzanti IAS, ma a minor chiarimenti nelle segnalazioni dei conti, a differenza da quelle richieste utlizzanti IAS.

In mancanza delle particolari IAS per le medie e le piccole imprese, gli Stati membri possono permettere, che queste imprese utilizzino i metodi della misurazione e della valutazione di IAS, ma a dare meno informazione nelle note dei conti di quelle richieste da IAS.

Ciò nonostante, le opinioni espresse per le modifiche delle direttive contabili continuano a essere non definitive per lo più, nella fase di discussione e delle proposte fatte per le modifiche, conoscenza, impossessamento e assimilizaione di queste disposizioni dalla maggior parte degli specialisti operanti nel settore della finanza e della contabilità, ne penso che si sia ritardato, se va tenuto presente il tempo dell'unificazione delle regole contabili nel continente che è vicino e noi questo tempo ci deve trovare preparati.

\section{L'approvazione delle IFRS (International Financial Reporting Standards ) per il Rapporto Finanziario delle Società Listate della U.E., Passo Indispensabile Verso la Convergenza Contabile}

Gli ultimi anni la Commissione degli Standards Internazionali della contabilità (IASB) (International Accounting Standards Board) ha visibilmente migliorato IFRS - facendo in questo modo grandi passi per ottenere la convergenza contabile, globale. Come si sa, cominciando dal mese gennaio 2005, tutte le società listate al livello U.E. (circa 7000 tali) sono state obbligate a preparare i loro conti consolidati ( i prospetti finanziari annui ) basati nell'IFRS. Oltre a questo sono stati tanti i paesi non europei, come per esempio I'Australia, Hong Kong, Israele, Nuova Zelanda, il Canada ecc., che hanno fatto convergere interamente o parzialmente i loro standards nazionali con l'IFRS.

Negli Stati Uniti d'America il supporto per la convergenza ha segnato crescite stabili. L'accordo di "The Norwalk Agreement" dell'anno 2002 tra FASB (Financial Accounting Standards Board) e IASB (International Accounting Standards Board) ha formalizzato l'impegno delle due commissioni per lavorare alla convergenza degli standards del rapporto finanziario. Secondo questo accordo questi due organismi si sono impegnati a fare sforzi maggiori a rendere i loro standard esistenti del rapporto fnanziario interamente comparabile e di coordinare i loro programmi di lavoro futuro per assicurare, che il fatto che siano comparabili, dopo che si sia ottenuto, andrà conservato anche nel futuro. Da quando è stato sottoscritto l'accordo sono statti fatti passi importanti. Così FASB ha pubblicato alcuni standards, che eliminano le differenze con IFRS e si presentano piuttosto convergenti con IFRS. FASB e IASB stanno lavorando insieme per compilare gli stessi standards in acuni campi principali, com'è quello della combinazione delle imprese, delle entrate, gli affitti obbligati e il rapporto della performance finanziaria dalle entità. Un progetto molto importante in corso, è anche il progetto per la compilazione di un quadro concettuale comune, che realizzerà miglioramenti e chiarimenti in merito alla conoscenza degli elementi dei prospetti finanziari. D'altronde anche IASB ha modificato alcuni dei suoi standards, presentandoli nella stessa linea con US GAAP (Generally Accepted Accounting Principles, United States ).

Così un ultimo esempio è la pubblicazione nel mese di gennaio 2006 di un progetto-presentazione, che ha proposto contemporaneamente le stesse richieste al rapporto segmentare di IASB con SFAS 131 (Statement of Financial Accounting Standards) (edito da FASB) esigendo l'adozione del metodo di direzione. Partito dalla necessità di fare molte modifiche inerenti alla converegenza con US GAAP e specialmente, per dare tempo alle società europee di applicare e consolidare l'utilizzzo di IFRS, nel luglio del 2006, IASB ha deciso, che nel mese di gennaio del 2009, a non permettere nessuna entrata in vigore di nessuna modifica considerevole fatta negli standards internazionali del rapporto finanziario. Comunque il lavoro di IASB e FASB seguirà con la stessa intensità anche durante questo periodo. II processo della convergenza contabile a livello di U.E. include l'adozione di IFRS, e le loro modalità di applicazione dalle società europee. La regolazione della Commissione Europea 1610612002 richiede che tutte le società listate nella U.E. preparino i prospetti finanziari consolidati in concomitanza con IFRS accettate da U.E. il 1 gennaio del 2005. La migliore supposizione dei seguaci della convergenza presuppone, che i paesi membri adottino per tutte le loro società, IFRS, come pure esse sono state pubblicate da ISAB (dunque senza modifiche). Se tutti i paesi membri della U.E. adottassero IFRS come i loro standards nazionali allora le società americane aventi filiali nei paesi U.E. non hanno bisogno di preparare i loro prospetti finanziari secondo i registri contabili molteplici. La decisione di U.E. per richiedere l'applicazione di IFRS per i prospetti finanziari consolidati delle società listate, fu un evento importante nella via della convergenza, benché essa non comandi interamente entro i paesi membri. Questo, perché la regolazione 160612002 permette a ognuno dei 25 paesi membri di stabilire da solo, se IFRS saranno richieste 0 permesse a esere utilizzate nella preparazione dei prospetti finanziari individuali della società listate o dei prospetti consolidati e di quelli individuali delle società non listate. Questo ha reso possibile, che solo alcuni Stati abbiano fatto scelte tra le suddette alternative, mentre 
gli altri no. (Nel maggio del 2006, 10 Stati membri avevano deciso l'utilizzo di IFRS per i prospetti finanziari individuali delle società listate, mentre solo 3 Stati vevano deciso come esigenza l'utilizzo di IFRS per i prospetti finanziari consolidati delle società non listate).

Volendo, che la contabilità la teniamo orientata sulle tassazioni, nei paesi di U.E. si sta osservando un sistema di rapporto nei due standards, rispettivamente il rapporto basato nei IFRS e quello basato negli standards nazionali di ogni paese.

\section{La Modernizzazione nelle Direttive 4 e 7 di C.E}

La direttiva della modernizzazione contabile (2003|51|EC), che ammenda la quarta direttiva sui conti annui individuali e la settima direttiva sui conti consolidati, (direttive, che hanno trovato una ampia trattazione nello suddetto scritto "le Direttive della Contabilità di U.E. e la loro evoluzione ", mira a portare le richieste contabili di U.E. nella stessa linea con la teoria e la pratica moderna contabile. In quegli Stati, ove non viene richiesta l'applicazione di IFRS per tutte le società, la finalità è I'orientamento delle società verso un rapporto similare e molto qualitativo. Dopo l'adozione di IFRS per le grandi società a livello di U.E., attenzione si sta dando alle società non listate e specialmente alle medie e piccole entità. In tutto il vecchio continente si calcolano pià di 23 milioni di medie e piccole imprese (SME), che sono molto importanti per l'economia europea. Le loro regole contabili si esige, che riflettano la natura della loro attività. Le SME devono avere regole dirette e forti e non vanno caricate eccessivamente con troppe richieste per dare le informazioni esplicative, che non hanno a che vedere con la natura delle attività, che esse svolgono.

Secondo il commissionario del mercato interno il signor Charlie McCreevy la U.E. sta lavorando per identificare quei campi della contabilità e della legge per le società commerciali della U.E., che per le SME devono essere semplificate. Secondo lui un lavoro molto importante per la contabilità e il rapporto finanziario delle SME si sta facendo anche dalla Commissione degli Standards Internazionali della Contabilità (IASB). Inoltre lui accentua che "alla Commissione è stato reso chiaro, che questo lavoro sarà utile, se essa mirerà la semplificazione". Dunque un lavoro utile in merito ai IFRS per le SME, per cui a dire il vero va detto, che costituisce una questione, che ha suscitato un interesse globale, incluse qui le Organizzazioni internazionali quali la Federazione Internazionale dei contabili (IFAC) (International Federation of Accountants), la banca Mondiale, U.E., FEE (European Federation of Accountants), e molti altri attori internazionali sarà il test pià chiaro per il loro utilizzo

( l'adozione) dalla U.E. anche su scala internazionale.

Come conclusione e in fine della informazione molto ampia data, per tutte e due direttive contabili 4 e 7 della C.E., a uno scopo preciso, il rapporto finanziario comparato a un'economia aperta e globale, dove i confini saranno sempre più convenzionali.

\section{References}

Charlie McCreevy 6 Nov 2008 Etat du secteur immobilier dans I'Union européenne

Discussion paper on the modernization of accounting directives.

Edition of European Federation of Accounting Experts, Brussels, April, 2001

Materials for internal discussions in the modernizations of accounting directives.

The Fourth Directive 78/660 of C.E. for all the annual accounts.

The General Accounting Plan, edition of the Ministry of Finance, Tirana 1993.

The National Standards of Accounting - Edition of National Council of Accounting, Tirana, 2006

The Seventh Directive nr. 831349 of C.E. for all the consolidated accounts. 
